\title{
わずかな曲率をもつ棒状分子溶液の固有粘度*
}

\author{
西 村二 郎** 岡 \\ by \\ Jiro Nishimura \\ (Central Research Laboratory, Showa Denko K.K., Tokyo) \\ and Syoten OkA \\ (Faculty of Science, Tokyo Metropolitan University, Tokyo)
}

小 天***

\section{Intrinsic Viscosity of a Solution Containing Slightly Curved Rod-Like Molecules}

As a preparative step to treat the intrinsic viscosity of a stiff chain macromolecule in a solution, we have investigated the hydrodynamic behavior of a slightly curved rigid rod in a shear field.

Regarding the longitudinal axis of the rod as a differentiable space curve, we can represent the curve as follows using the Bouquet's formula : $\boldsymbol{r}(s)=\boldsymbol{r}^{(0)}(s)+\kappa_{1} \boldsymbol{r}^{(1)}(s)+\kappa_{1}{ }^{2} \boldsymbol{r}^{(2)}(s)+\kappa_{1}^{\prime} \boldsymbol{r}^{(3)}(s)+\kappa_{1} \kappa_{2} \boldsymbol{r}^{(4)}(s)+$ $\ldots$, where $\kappa_{1}$ and $\kappa_{2}$ are the curvature and the torsion of the curve at the middle point of it and the prime stands for the differentiation with respect to $s$, the contour length of the curve. The force distribution $\boldsymbol{F}$ on the longitudinal axis when the rod is in the shear field can be estimated by the Oseen's method : $\frac{1}{8 \pi \mu} \int_{-a}^{a} T\left(s, s_{0}\right) \cdot \boldsymbol{F}(s) d s=-\boldsymbol{V}_{r}\left(s_{0}\right)$, where $a$ is the half length of the rod, $\mu$ the viscosity of the fluid, $T$ the interaction tensor and $V_{r}$ is the relative velocity to the rod. The Brownian motion is not taken into account here. Expanding $\boldsymbol{T}, \boldsymbol{F}$ and $\boldsymbol{V}_{r}$ into power series of $\left(\kappa_{1}, \kappa_{1}{ }^{\prime}, \cdots ; \kappa_{2}, \kappa_{2}{ }^{\prime}, \cdots\right)$, we can obtain a set of equations instead of the above equation. The first equation of the set is identical with that for the straight rod derived by Burgers. The motion of the rod is to be determined upon the conditions of the balance of the force and of the moment of force.

Concrete calculation is made of a rod curved like a circular arc, and the intrinsic viscosity for the case is estimated as follows :

$$
[\mu]=\frac{2}{3 \pi \rho} \frac{a}{b} \frac{1}{\sigma-1.80} \sqrt{1+\left(\frac{a}{b}\right)^{2}\left(\kappa_{1} a\right)^{2} \frac{\sigma-2.13}{10(\sigma-1.80)}},
$$

where $\rho$ and $b$ are the density and the radius of the cross-section, of the rod and $\sigma=\log 2 a / b$.

(Received Feb. 28, 1967)

\section{1 ま が き}

高分子の中には近似的にわずかな届曲性をもつ棒 状粒子と考えられるものがある。このような型の高 分子の諸性質の理論的取り扱いに関しては, 斉藤らや Hearst らの研究が有用であろう。しかし，てのよう な分子の溶液の固有粘度については, 充分に論議が尽 くされているとは思えないようである。こてではその ような議論の第一段階として，わずかに曲がった棒状 粒子が剛体としてずり速度場中に存在するときの挙動 と流れの場の乱れを計算し，合わせてての場合の固有 粘度を評価するてとを試みた。

* 原稿受理 昭和 42 年 2 月 28 日

** 正会員 㫝和電工(株) 中央研究所 東京都大田区原盯

*** 正会 員 身京都立大学理学部 東京都世田谷区深㐸䢹 1 丁月
真直な棒状粒子小場合については Burgersの部算が よく知られている，粒子が曲がっている場合粒子の中 心軸（=空間曲線）は, Bouquet の公式によって曲線 上の定点からの長さのべき級数で表わされる，ての表 現とOseenの方法とを組み合わせると， Burgers の “真直な棒”の場合に準ずる積分方程式が一連得られる. てれを遂次解いていけば“曲がり”の影響が付加され た項を次々と得ることができる。

初めに計算方法について一般的に考光, 後で円弧状 粒子について実際に計算を行なった。なお，簡単のた め非常に紐長い粒子を取り上げた。 また粒子の慣性を 無視し,さらに Brown 運動も考慮に入れていない.

\section{2 粒子の形状の規定}

いま,一つの静止座標系を $O-X Y Z$ とし, 粒子が存 
在しないとき $\boldsymbol{U}=(q Y, 0,0)$ なる流れの場があるも のとする．次に，その流れの場の中に存在する任意の 粒子に着目し，その重心 $G$ を原点とし静止系と平行 移動の関係にある座標系 $o-x y z$ を導入する。 さらにこ の重心座標之原点を同じくし，粒子の中心軸を表わす 曲線の中点 $M$ における切線, 法線および従法線方向 を三軸とする曲線座標系（と名づける） $0-\xi \eta ら$ と考元 る. 点 $G$ の静止系们ける位置を $\left(X_{G}, Y_{G}, Z_{G}\right)$ と し，また，重心系 $\rightarrow$ 曲線系の Euler の角を $(\phi, \theta, \phi)$ とすると各座標系間の変換は次の式で与えられる。

$(x, y, z)=(X, Y, Z)-\left(X_{G}, Y_{G}, Z_{G}\right)$

$(\xi, \eta, \zeta)^{T}=A \cdot(x, y, z)^{T}$

ただし

$A=\left[\alpha_{\imath}\right]$

$=\left(\begin{array}{ccc}1 & 0 & 0 \\ 0 & \cos \psi & \sin \psi \\ 0 & -\sin \phi & \cos \psi\end{array}\right)\left(\begin{array}{ccc}\cos \theta & 0 & -\sin \theta \\ 0 & 1 & 0 \\ \sin \theta & 0 & \cos \theta\end{array}\right)\left(\begin{array}{ccc}1 & 0 & 0 \\ 0 & \cos \phi & \sin \phi \\ 0 & -\sin \phi & \cos \phi\end{array}\right)$

なお指標 $T$ は転置行列を表わす。

点 $M$ から曲線に沿って測った長さを $s$ とすると， 曲線は Bouguet の公式を用いて次のように表わされ る。

$$
\begin{aligned}
\boldsymbol{r}(s) & =\left(\begin{array}{l}
\xi \\
\eta \\
\zeta
\end{array}\right)=\left(\begin{array}{l}
s-\frac{1}{6} \kappa_{1}{ }^{2} s^{3}-\frac{1}{8} \kappa_{1} \kappa_{1}{ }^{\prime}\left(s^{4}-\frac{1}{5} a^{4}\right)+\cdots \\
\frac{1}{2} \kappa_{1}\left(s^{2}-\frac{1}{3} a^{2}\right)+\frac{1}{6} \kappa_{1}{ }^{3}+\cdots \\
\frac{1}{6} \kappa_{1} \kappa_{2} s^{3}+\cdots
\end{array}\right) \\
& =\boldsymbol{r}^{(0)}+\kappa_{1} \boldsymbol{r}^{(1)}+\kappa_{1}{ }^{2} \boldsymbol{r}^{(2)}+\kappa_{1}{ }^{\prime} \boldsymbol{r}^{(3)}+\kappa_{1} \kappa_{2} \boldsymbol{r}^{(4)}+\cdots
\end{aligned}
$$

ただし $\kappa_{1}, \kappa_{2}$ は点 $M$ における曲線の曲率，㨭率を 表わし，プライムはそれらの $s$ に関する微分を意味 する。 また， $2 a$ は粒子の長さであり， $-a \leq s \leq b$ であ る.

\section{3 流れの場の乱れ}

曲線上の線素 $[s, s+\Delta s]$ 亿働く力 $\boldsymbol{F}(s) \Delta s$ による流 れの乱れは Oseen の方法によって評価するてとがで きる. 曲線上の点 $s=s_{0}$ における粒子の表面での乱れ が，粒子表面に相対的な流れを，断面の円周上で “平 均的”に打消すととを要求すれば次の上うな式がなり たつ。

$$
\begin{aligned}
& \frac{1}{8 \pi \mu} \int_{-a}^{a}\left[T^{(0)}\left(s, s_{0}\right)+\kappa_{1} T^{(1)}+\kappa_{1}{ }^{2} T^{(2)}+\kappa_{1}{ }^{\prime} T^{(3)}+\cdots\right] \\
& \boldsymbol{F}(s) d s=-\boldsymbol{V}_{r}\left(s_{0}\right) \\
& \text { ただし, } \boldsymbol{r}_{0}=\sqrt{\left(s-s_{0}\right)^{2}+b^{2}} \\
& T^{(0)}=\left(\begin{array}{ccc}
\frac{2}{r_{0}}-\frac{b^{2}}{r_{0}{ }^{3}} & 0 & 0 \\
0 & \frac{1}{r_{0}}+\frac{1}{2} \frac{b^{2}}{r_{0}^{3}} & 0 \\
0 & 0 & \frac{1}{r_{0}}+\frac{1}{2} \frac{b^{2}}{r_{0}^{3}}
\end{array}\right),
\end{aligned}
$$

$T^{(1)}=\left(\begin{array}{ccc}0 & \frac{1}{2} \frac{1}{r_{0}{ }^{3}}\left\{\left(s-s_{0}\right)^{2}\left(s+s_{0}\right)-b^{2} s_{0}\right\} & 0 \\ * & 0 & 0 \\ 0 & 0 & 0\end{array}\right), \cdots .(7)$

$\mu$ は流体の粘度， $\boldsymbol{b}$ は粒子の断面の半径である。また， $V_{r}$ は粒子の表面に対する相刘速度である。

$\boldsymbol{F}(s), \boldsymbol{V}_{1}(s)$ を $\left(\kappa_{1}, \kappa_{1}{ }^{\prime} \cdots ; \kappa_{2}, \kappa_{2}{ }^{\prime} \cdots\right)$ で展開する.

$$
\begin{gathered}
\boldsymbol{F}=\boldsymbol{F}^{(0)}+\kappa_{1} \boldsymbol{F}^{(1)}+\kappa_{1}{ }^{2} \boldsymbol{F}^{(2)}+\kappa_{1}{ }^{\prime} \boldsymbol{F}^{(3)}+\cdots \\
\boldsymbol{V}_{r}=\boldsymbol{V}_{r}^{(0)}+\kappa_{1} \boldsymbol{V}_{\boldsymbol{r}}^{(1)}+\kappa_{1}{ }^{2} \boldsymbol{V}_{r}^{(2)}+\kappa_{1}{ }^{\prime} \boldsymbol{V}_{r}^{(3)}+\cdots
\end{gathered}
$$

(8)，(9)を(5)式に代入して両辺を比較すれば，

$$
\frac{1}{8 \pi \mu} \int_{-a}^{a} T^{(0)}\left(s, s_{0}\right) \cdot \boldsymbol{F}^{(k)}(s) d s=-\boldsymbol{G}^{(k)}\left(s_{0}\right)
$$$$
\left(k=1,2, \cdots ; \boldsymbol{G}^{(0)}=\boldsymbol{V}_{r}^{(0)}\right)
$$

が得られる。ただし，

$$
\boldsymbol{G}^{(k)}=\boldsymbol{V}_{r}(k)+\frac{1}{8 \pi \mu} \int_{-a}^{a} \sum_{\imath, \jmath=0}^{\imath+\jmath=k} T^{(\imath)} \cdot \boldsymbol{F}^{(\jmath)} d s . \quad \text { (11) }
$$

積分方程式(10)の核となっている関数をデル夕関数を用 いて次のように大胆に近似する。

$$
\frac{a}{r_{0}}=2(\sigma-1.30) \delta\left(s-s_{0}\right) ; \frac{a b^{2}}{r_{0}{ }^{3}}=2 \delta\left(s-s_{0}\right) \quad \text { (12) }
$$

ただし， $\sigma=\log 2 a / b$ とおいた。 上式中の定数值は, 曲率がない場合のエネルギ損失の值が Burgers の計 算と一致することおよび基準化の条件から定めた。 のとき(10)式は容易に解ける。

$$
\begin{aligned}
& F_{\xi}^{(k)}=-\frac{2 \pi \mu}{\sigma-1.80}\left(G_{\xi}^{(k)}-\frac{1.30}{\sigma-1.80} \cdot \bar{G}_{\xi}^{(k)}\right) \\
& F_{\nu}(k)=-\frac{4 \pi \mu}{\sigma-0.80}\left(G_{\nu}(k)-\frac{1.30}{\sigma+0.50} \cdot \bar{G}_{\nu}(k)\right) \nu \epsilon(\eta, \zeta)
\end{aligned}
$$

ただし, $\boldsymbol{F}^{(k)}=\left(F_{\xi}^{(k)}, F_{\eta}^{(k)}, F_{\xi}^{(k)}\right)^{T}, \boldsymbol{G}^{(k)}=\left(G_{\xi}(k)\right.$, $\left.G_{\eta}^{(k)}, G_{\zeta}^{(k)}\right)^{T}$ であり，また $\bar{G}_{\xi}^{(k)}=\frac{1}{2} \int_{-1}^{1} G_{\xi}^{(k)}(u) d u$ $\left(u=\frac{s}{a}\right)$ である.

\section{4 粒子の挙動}

相刘速度 $\boldsymbol{V}_{\boldsymbol{r}}$ は次の式で与兄られる。

$$
\boldsymbol{V}_{r}=(q B-\Omega) \cdot \boldsymbol{r}-A \cdot\left(\boldsymbol{U}_{G}-\boldsymbol{U}^{(0)}\right)
$$
ただし，

$$
B=\left\{\beta_{\imath \jmath}\right]=\left\{\alpha_{\imath 1} \alpha_{\jmath 2}\right] ; \Omega=\left(\begin{array}{lcc}
0 & -\omega_{\zeta} & \omega_{\eta} \\
\omega_{\zeta} & 0 & -\omega_{\xi} \\
-\omega_{\eta} & \omega_{\xi} & 0
\end{array}\right)
$$

また, $\boldsymbol{U}^{(0)}=\left(q Y_{G}, 0,0\right)^{T}$ と $\boldsymbol{U}_{G}=\left(U_{G}, V_{G}, W_{G}\right)^{T}$ は静止系に関する，点 $G$ における元の流れの速度と 点の移動速度を, $\boldsymbol{\omega}=\left(\omega_{\xi}, \omega_{\eta}, \omega_{\xi}\right)$ は粒子の重心の囲 りの角速度を表わす。 $U_{G}$ と $\omega$ はそれぞれ力のつり あいと力のモーメントのつりあいから定めることがで きる。すなわち，

$$
\int_{-1}^{1} \boldsymbol{F}(u) d u=0 ; \int_{-1}^{1} \boldsymbol{r}(u) \times \boldsymbol{F}(u) d u=0
$$

$\boldsymbol{\omega}, \boldsymbol{U}_{G}, A, B$ を(8)または(9)式のような形で表わすと(14) 
式は， $\boldsymbol{U}^{(j)}=0(j \neq 0)$ として次のようになる.

$\boldsymbol{V}_{r}^{(k)}=\sum_{\imath, \jmath=0}^{\imath+\jmath=k}\left\{\left(q B^{(\imath)}-\Omega^{(\imath)}\right) \cdot \boldsymbol{r}^{(j)}-A^{(\imath)} \cdot\left(\boldsymbol{U}_{G}^{(\jmath)}-\boldsymbol{U}^{(\jmath)}\right)\right]$

ここで一般的には， $\boldsymbol{U}_{G} \neq \boldsymbol{U}^{(0)}$ なることに注意してお

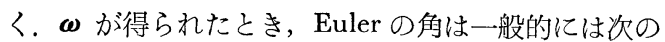
式から定めるてとができる。

$\frac{d}{d t}\left(\begin{array}{c}\phi \\ \theta \\ \phi\end{array}\right)=\left(\begin{array}{ccc}0 & \operatorname{cosec} \theta \sin \psi & \operatorname{cosec} \theta \cos \phi \\ 0 & \cos \phi & -\sin \phi \\ 1 & -\cot \theta \sin \psi & -\cot \theta \cos \phi\end{array}\right) \cdot \omega(18)$ 中心軸が平面曲線の場合には粒子の太さの效果を別に 加味してやる必要がある.

\section{5 固 有 粘 度}

粒子の存在による, 単位時間, 単位体積あたりのエ ネルギ損失の増加は，粒子密度を $n$ として次式で与え られる。

$$
\begin{aligned}
\frac{d w}{d t} & =-a n \int_{-1}^{1}\left(\boldsymbol{V}_{r}^{(0)} \cdot \boldsymbol{F}^{(0)}+\kappa_{1} \sum_{\imath, j=0}^{\imath+\jmath=1} \boldsymbol{V}_{r}^{(i)} \cdot \boldsymbol{F}^{(j)}\right. \\
& \left.+{\kappa_{1}}^{2}{ }^{2+} \sum_{\imath, j=0}^{=2} \boldsymbol{V}_{r}^{(i)} \cdot \boldsymbol{F}^{(j)}+\cdots\right) d u
\end{aligned}
$$

固有粘度は次のとおりである.

$$
\lceil\mu\rceil=\frac{1}{q^{2} \mu n} \cdot \frac{1}{2 \pi \rho a b^{2}} \cdot \frac{d w}{d t}
$$

ただし， $\rho$ は粒子の密度である.

\section{6 円弧状粒子の場合}

粒子が円弧のごく一部であるような場合について上 述の方法を適用してみる。結果は下記のとおりである。

$$
\begin{aligned}
& \boldsymbol{\omega}^{(0)}=q\left(\left\{\alpha_{31} \alpha_{22}\right\}^{(0)},-\left[\alpha_{31} \alpha_{12}\right]^{(0)},\left\{\alpha_{21} \alpha_{12}\right\}^{(0)}\right)^{T}(21) \\
& \boldsymbol{U}_{G}^{(0)}=\left(q Y_{G}, 0,0\right)^{T} \\
& \boldsymbol{F}^{(0)}=\left(-\frac{2 \pi \mu}{\sigma-1.80} q a\left[\alpha_{11} \alpha_{12}\right]^{(0)}, 0.0\right)^{T} \\
& \boldsymbol{\omega}^{(1)}=q\left(\left[\alpha_{31} \alpha_{22}\right]^{(1)},-\left[\alpha_{31} \alpha_{12}\right\}^{(1)},\left\{\alpha_{21} \alpha_{12}\right\}^{(1)}\right)^{T} \text { (24) } \\
& \boldsymbol{U}_{G^{(1)}}=-\frac{1}{6} \frac{\sigma-2.15}{\sigma-1.80} q a^{2}\left(\left\{\alpha_{11} \alpha_{12} \alpha_{21}\right]^{(0)},\right. \\
& \left.\left\{\alpha_{11} \alpha_{12} \alpha_{22}\right\}^{(0)},\left[\alpha_{11} \alpha_{12} \alpha_{22}\right\}^{(0)}\right)^{T} \\
& \boldsymbol{F}^{(1)}=\left(-\frac{2 \pi \mu}{\sigma-1.80} q a\left[\left(\alpha_{11} \alpha_{12}\right)^{(1)} u+\frac{1}{2} a\left(\alpha_{11} \alpha_{22}\right.\right.\right. \\
& \left.\left.+\alpha_{21} \alpha_{12}\right)(0)\left(u^{2}-\frac{1}{3}\right)\right],-\frac{2 \pi \mu}{\sigma-0.80} q a^{2}\left[\alpha_{21} \alpha_{22}\right. \\
& \left.\left.-\frac{\sigma-2.80}{\sigma-1.80} \alpha_{11} \alpha_{12}\right]^{(0)}\left(u^{2}-\frac{1}{3}\right), 0\right)^{T} \\
& \boldsymbol{\omega}^{(2)}=q\left(\left[\alpha_{31} \alpha_{22}\right]^{(2)},-\left\lceil\alpha_{31} \alpha_{12}\right]^{(2)},\left[\alpha_{21} \alpha_{12}\right\rfloor^{(2)}\right. \\
& \left.-\frac{1}{10} a^{2} \frac{\sigma-2.13}{\sigma-1.80}\left\{\alpha_{11} \alpha_{22}+\alpha_{21} \alpha_{12}\right\}^{(0)}\right)^{T}
\end{aligned}
$$

さて, 上式で $\omega=\omega^{(0)}+\kappa_{1} \omega^{(1)}+\kappa_{1}^{2} \omega^{(2)}$ など作って みると, $\left(\alpha_{\imath \jmath} \cdots\right)$ の右肩の記号が整理される。たとば， $\omega_{\xi} \doteqdot q\left\lceil\left(\alpha_{31} \alpha_{22}\right]^{(0)}+\kappa_{1}\left(\alpha_{31} \alpha_{22}\right)^{(1)}+\kappa_{1}{ }^{2}\left(\alpha_{31} \alpha_{22}\right)^{(2)}\right\}$

$\fallingdotseq q \alpha_{31} \alpha_{22}$

のとおりである．とのととに注意しながらエネルギ損 失の堌加を諳算すると次のような式が得られる。

$$
\begin{aligned}
\frac{d w}{d t} & =q^{2} a^{3}\left[\frac{4 \pi \mu}{3(\sigma-1.80)}\left(\alpha_{11} \alpha_{12}\right)^{2}\right. \\
& \left.+\left(\kappa_{1} a\right)^{2}\left\{\frac{4 \pi \mu}{45(\sigma-1.80)}\left(\alpha_{11} \alpha_{22}+\alpha_{21} \alpha_{12}\right)^{2}+\cdots\right\}+\cdots\right]
\end{aligned}
$$

$\left(\alpha_{11} \alpha_{12}\right)^{2}$ などの時間平均を求めるには次の微分方程式 を解かなければならない。

$\dot{\phi}=-q \varepsilon^{2} \cos \phi \cot \theta+\left(\kappa_{1} a\right)^{2} q \lambda \operatorname{cosec} \theta \cos \phi \quad$ (29)

$\dot{\theta}=-q\left\lceil\left(1-\varepsilon^{2}\right) \sin ^{2} \theta+\varepsilon^{2}\right\rceil \sin \phi-\left(\kappa_{1} a\right)^{2} q \lambda \sin \phi \quad(30)$

$\dot{\psi}=q \sin \theta \cos \phi(\cos \phi \cos \phi-\sin \phi \cos \theta \sin \phi)$

$-\left(\kappa_{1} a\right)^{2} q \lambda \cot \theta \cos \psi$

(31)

ただし， $\varepsilon=\frac{b}{a} ; \lambda=\frac{1}{10} \frac{\sigma-2.13}{\sigma-1.80}(\cos \phi \cos \theta \cos \psi$

$-\sin \phi \cos 2 \theta \sin \phi)$.

上式で初めの 2 式は(18)式に太さの効果を加味したもの である.すなわち $\kappa_{1}=0$ とおいたものは, Jeffery の 偏長回転だ円体の場合と等価な式になっている。上記 3 式においてまず $\kappa_{1}=0$ の場合の解を求め, その值を 各式の右辺の第 2 項に代入してふたたび $\phi, \theta$ を定め る. その結果から, $\left(\alpha_{11} \alpha_{12}\right)^{2}$ の時間平均を計算すると 次のようになる.

$$
<\left(\alpha_{11} \alpha_{12}\right)^{2}>=\frac{1}{\pi} \sqrt{\varepsilon^{2}+\left(\kappa_{1} a\right)^{2} \frac{\sigma-2.13}{10(\sigma-1.80)}}
$$

この式から明らかなように，根号の中の第 2 項が $\varepsilon^{2} に$ 比べて著しく小さくない限り, (28)式の大カッコの中の 第 2 項以下は第 1 項に比べて無視するてとができる. 結局国有粘度は次のようになる。

$$
\begin{gathered}
\lceil\mu]=\frac{2}{3 \pi \rho} \frac{a}{b} \frac{1}{\sigma-1.80} \sqrt{1+\left(\kappa_{1} a\right)^{2}\left(\frac{a}{b}\right)^{2} \frac{\sigma-2.13}{10(\sigma-1.80)}} \\
7 \text { あ } \\
\text { よ がき }
\end{gathered}
$$

以上かなりわずらわしい誢を行なったのも，粒子 と流体の相互作用を, “Friction coefficient”という概 念で置き換えるととに刘し抵抗を感じたからにほかな らない，ての結果によれば，わずかな“曲がり”の影 響が意外に大きくきいている。 また粒子の重心が，粒 子の “非対称性”によって，一般に周囲の流れと異な る移動速度（特に流れの方向に上な成分）をもつとい う結果もなかなか興味深い.しかし，ててで得られた 固有粘度を, 実際の高分子溶液のものと対応させるに は，粒子に屈曲性を持たせること，Brown運動の效果 を考えるととなどが必要になってくるものと思われる。

(炤和141年11月26日 第15回レオロノ一討論会にて講演)

\section{参 考 文 献}

1）齐藤信彦，高橋邦彦，第21回日本物理学会年会滥 演予稿集 2, p. 37 (1966).

2) Harris, R.M., and J.E. Hearst, J. Chem. Phys., 44, 2595 (1966).

3) Burgers, J.M., "Second Report on Viscosity and Plasticity", Chap. 3 (1938) Amsterdam. 